\title{
Combined coherent radar/lidar system on chip
}

\section{Antonella Bogoni, Fabio Falconi, Muhammad Malik, Micro Scaffardi, Claudio Porzi, et al.}

Antonella Bogoni, Fabio Falconi, Muhammad Malik, Micro Scaffardi, Claudio Porzi, Filippo Scotti, Giorgia Parca, Luigi Ansalone, Paolo Ghelfi, "Combined coherent radar/lidar system on chip," Proc. SPIE 11852, International Conference on Space Optics - ICSO 2020, 118526G (11 June 2021); doi: $10.1117 / 12.2600290$

SPIE Event: International Conference on Space Optics - ICSO 2021, 2021, Online Only 


\section{International Conference on Space Optics-ICSO 2020}

Virtual Conference

30 March-2 April 2021

Edited by Bruno Cugny, Zoran Sodnik, and Nikos Karafolas
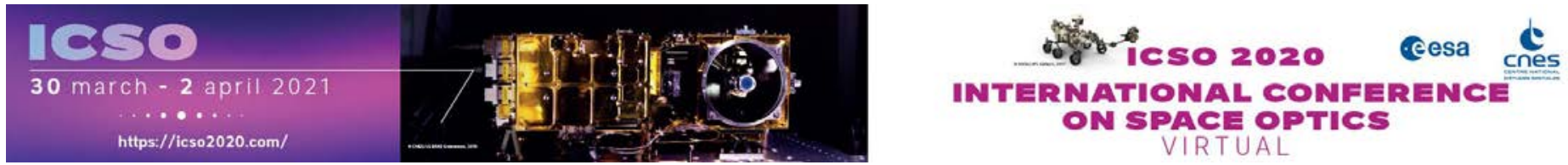

\section{Combined coherent radar/lidar system on chip}

\section{Cesa isopmeatians ecnes}




\title{
Combined coherent radar/lidar system on chip
}

\author{
Fabio Falconi ${ }^{(\mathrm{a})}$, Muhammad Malik ${ }^{(\mathrm{a})}$, Mirco Scaffardi $^{(\mathrm{b})}$, Claudio Porzi ${ }^{(\mathrm{a})}$, Filippo Scotti $^{(\mathrm{b})}$, \\ Giorgia Parca $^{(\mathrm{c})}$, Luigi Ansalone ${ }^{(\mathrm{c})}$, Paolo Ghelfi ${ }^{(\mathrm{b})}$, Antonella Bogoni ${ }^{(\mathrm{a}, \mathrm{b})}$ \\ a'Scuola Superiore Sant'Anna - TeCIP institute, Via Moruzzi 1, 56124 Pisa, Italy \\ ${ }^{\mathrm{b}}$ CNIT - National Photonic Networks Laboratory, Via Moruzzi 1, 56124 Pisa, Italy \\ ${ }^{c}$ ASI - Italian Space Agency, Via del Politecnico snc, 00133 Roma, Italy
}

\begin{abstract}
This work reports the first realization of a combined radar\&lidar system based on silicon-on-insulator photonic integrated circuit (PIC). The software-defined architecture comprises a frequency-flexible and simultaneous multi-band radar operation and RF communications, and an advanced lidar with coherent detection for range and velocity measurements and optical communications. Both systems are implemented within a single chip, allowing a coherent radar and lidar parallel data acquisition in order to take advantage of their complementary characteristics. Moreover, radar and lidar subsystems are able to share the same optical source and digital electronics unit, allowing for an ultimate reduction of size, weight, and power consumption, thus making the proposed architecture suitable for the most stringent applications.
\end{abstract}

Keywords: Coherent radar, coherent lidar, photonic integrated circuits, microwave photonics

\section{INTRODUCTION}

Radar (radio detection and ranging) and lidar (light detection and ranging) systems are both sensing technologies that exploit the back scattered energy - in the radio and optical domain. Radar systems are largely adopted since they can operate in all-weather conditions. Lidar systems, on the other hand, have superior angular resolution, but they are strongly affected by the weather and atmospheric attenuations, which ultimately limit their range. Radars and lidars therefore show complementary features, which makes desirable realizing a combined radar\&lidar system in order to take advantage of the best features of each system in a single architecture, ultimately providing a more complete and precise analysis of the area under observation.

Recently, photonics has proven to be a powerful tool for the development of RF and optical transceiver for radar and lidar systems. In fact, photonics has been successfully applied in the generation and detection of microwave signals providing a complete multiband photonics-based radar system [1]. Moreover, integrated photonic technologies have very recently been used to implement photonics-based radars with largely reduced size, weight, and power consumption (SWaP) [2] thus enabling robust and promising solutions for space applications where SWaP reduction is a crucial factor. On the other hand, lidar systems are natively photonic systems, and SOI devices have been already explored [3]. Therefore, it is possible to combine the radar and lidar functionalities in a unique photonics-based system exploiting their complementary characteristics [4]. Additionally, a combined radar\&lidar system can provide heterogeneous data captured in uniform detection conditions, which is a very important feature for precise data fusion implementation.

Here we propose a combined radar\&lidar system, in which the RF circuit of the radar transceiver responsible for the upand down-conversions has been replaced by an optical structure, and part of that structure is shared with the lidar transceiver. This hybrid system potentially simplifies the combination of the data detected by the radar and lidar sensors due to their intrinsic simultaneity, avoiding the synchronization process and resulting in a more precise and timely final imaging. Moreover, sharing the optical source also allows reducing the system cost and complexity. Thanks to the wide bandwidth of photonics, the architecture allows the implementation of the software-defined paradigm for both the radar and lidar functionalities (i.e., the RF and optical signals can be changed in waveform and frequency by reconfiguring via software the digitally-generated driving signals). Consequently, the radar and lidar transceivers can also be exploited for $\mathrm{RF}$ and optical communications. This results in a single multifunction ultra-compact integrated device, which is suitable 
for the most demanding platforms such as small payloads at LEO orbits and reduced processing complexity requirements.

In this paper we describe the first realization of a combined radar\&lidar and RF/optical communication system based on integrated photonic technology, developed within the project "RODI-RF/OPTICAL Combined coherent Transceiver for RADAR/LIDAR and RF/Optical communications in space" funded by the Italian Space Agency for the technological validation of photonic systems for space applications. The SOI platform is chosen for its large functionalities and technological maturity. The photonic integrated circuit (PIC) is designed for implementing i) a frequency-flexible and simultaneously multi-band radar, and ii) an advanced lidar with coherent photonic detection for velocity measurement.

\section{COMBINED RADAR \& LIDAR SYSTEM}

The combined radar\&lidar integrated photonics-based system has been developed on the SOI technological platform, because of its capability to integrate passive components, electro-optical modulators, and photodiodes with wellestablished maturity. Fig. 1 reports the architecture of the designed PIC, which has been realized through a multi-project wafer service. The inset shows a picture of the fabricated chip, with a total dimension of $5 \times 5 \mathrm{~mm}^{2}$. In the figure, the transmitter (TX) and receiver (RX) of both the lidar and radar subsystems are clearly recognizable. Moreover, the target emulators for both radar and lidar are also reported.

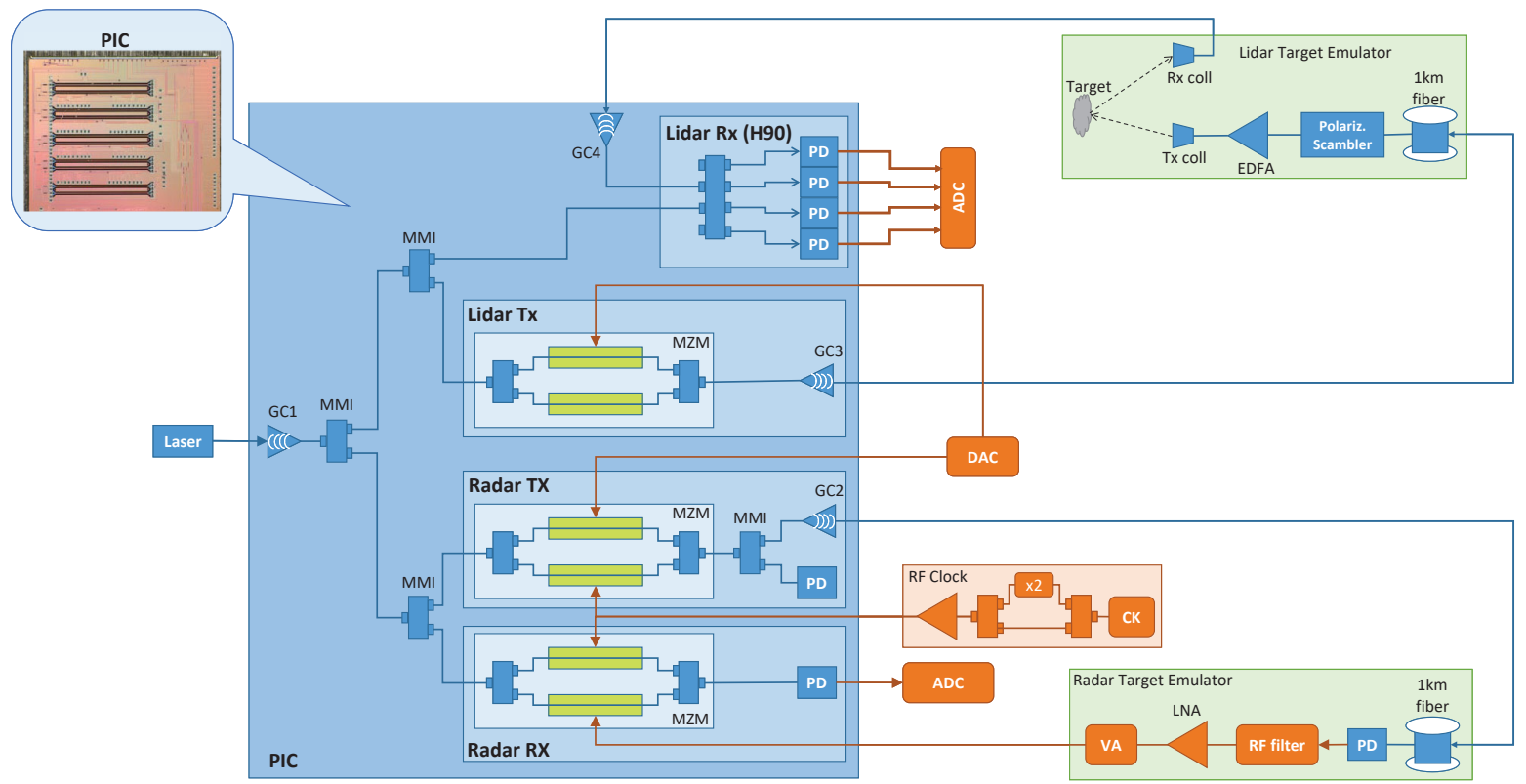

Figure 1. Architecture of the realized radar\&lidar transceiver, including the experimental setup implemented for system testing. GC: grating coupler, MMI: multimode interference splitter, MZM: Mach Zehnder modulator, PD: photodiode.

An external laser source is launched into the PIC through a vertical grating coupler (GC). After the GC, the input light is split by a $1 \times 2$ multimode interference splitter (MMI) to feed both the radar and lidar subsystems.

At the radar subsystem, the laser is first split by a 1x2 MMI to feed both the TX and the RX sections of the radar. The two sections are almost identical, consisting in a Mach Zehnder modulator (MZM) followed by a Si-Ge photodiode (PD). In the case of the TX, an MMI before the PD allows to monitor the optical signal out of the PIC through a GC for debugging purposes. In both the TX and RX, one arm of the MZM is driven by an high power RF clock signal in order to genere an optical comb within the phase-modulator of the MZM arm. In order to do so, a reference clock (CK) at frequency $\mathrm{f}_{\mathrm{CK}}$ is split into two replicas, with one of them frequency multiplied by 2 , and then recombined together. The obtained RF clock signal is composed by two tones at $\mathrm{f}_{\mathrm{CK}}$ and $2 \mathrm{f}_{\mathrm{CK}}$, thus helping the generation of higher order harmonics in the optical modulators. In fact, several optical modulation sidebands are generated inside the MZMs at an 
optical detuning of $\pm \mathrm{f}_{\mathrm{CK}}, \pm 2 \mathrm{f}_{\mathrm{CK}}, \pm 3 \mathrm{f}_{\mathrm{CK}}, \pm 4 \mathrm{f}_{\mathrm{CK}}$, etc. from the laser carrier frequency. In the TX MZM, the second arm is driven by signal at intermediate frequency (IF) $\mathrm{f}_{\mathrm{IF}}$, generated by a digital-to-analog converter (DAC) and consisting the pulsed radar waveform. At the TX PD, the optical components generated by the RF comb beat with the optical sidebands generated by the DAC, implementing the RF up-conversion of the IF signal to the multiples of the clock frequency, i.e. $f_{R F}=n f_{C K} \pm f_{I F}$. After the TX PD, specific RF front ends can eventually filter and amplify the selected up-converted radar signal. Similarly, in the RX MZM the received radar echo at $f_{R F}$ modulates the second arm of the MZM, and the RX PD implements the down-conversion to the original $\mathrm{f}_{\mathrm{IF}}$, so that the signal can be acquired by an analog-to-digital converter (ADC).

Similarly, the lidar subsystem is organized in a TX section and a RX section, both fed by the input laser source. The lidar TX section is consists in a single MZM that loads a modulation pattern on the optical signal, so that the range measure can be extracted from the crosscorrelation between the transmitted and received signals. The modulated optical signal is extracted from the PIC via a GC and sent to an optical frontend (an amplifier and a collimator) to be launched in the observed scene. The collected echo signal is coupled back into the PIC by another GC and routed in the lidar RX section. It is composed by a $90^{\circ}$ optical hybrid coupler (H90) that, by mixing the echo with the original unmodulated optical signal, implements a coherent detection. In fact, the H90 is fed by the original laser acting as optical local oscillator, and by the received optical echo, collected by an optical frontend, and the four outputs are detected by four PDs integrated in the PIC, allowing the digitization by a four-channel ADC. Subtracting the digitized signals two-by-two, the in-phase and quadrature components of the baseband received signal can be reconstructed and analyzed.

\section{CHARACTERIZATION OF THE RADAR SUBSYSTEM}

In order to characterize the radar subsystem, the PIC is fed by a $15 \mathrm{dBm}$ narrow linewidth continuous wave DFB laser. The reference clock signal is generated by a synthesizer at $\mathrm{f}_{\mathrm{CK}}=3.5 \mathrm{GHz}$, thus providing replicas also at $7 \mathrm{GHz}$, $10.5 \mathrm{GHz}$, and $14 \mathrm{GHz}$, allowing the system to operate up to the $\mathrm{Ku}$ band. The corresponding DAC is set to generate a plain radar pulse with duration and period of $2 \mu \mathrm{s}$ and $40 \mu \mathrm{s}$, respectively, centered at $\mathrm{f}_{\mathrm{IF}}=100 \mathrm{MHz}$. Moreover, all the electrical signals are loaded with a $3 \mathrm{~V}$ DC component in order to properly bias the p-n junctions composing the modulators. Fig. 2-A reports the RF spectra as measured at the radar TX PD and, as can be seen, clear replicas of the IF signal are generated at $3.4 \mathrm{GHz}$ ( $\mathrm{S}$ band), $6.9 \mathrm{GHz}$ (C band), $10.4 \mathrm{GHz}$ (X band), and $14.1 \mathrm{GHz}$ ( $\mathrm{Ku}$ band), confirming the capability of the PIC radar section to up-convert the radar signal to several carrier frequencies. The radar RX section implements the complementary function with similar performance.
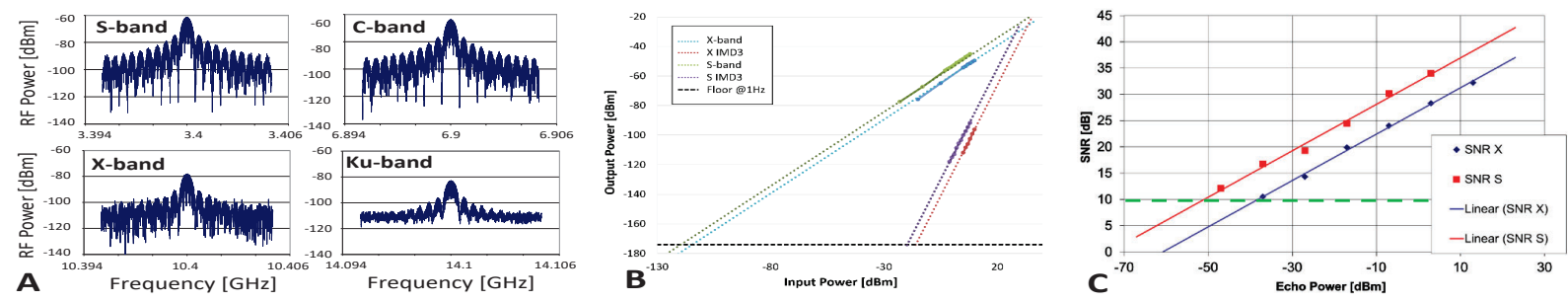

Figure 2. A) Radar signal generated in the S, C, X and Ku bands; B) Two-tone spurious frequencies analysis of the radar subsystem; C) Radar sensitivity in $\mathrm{S}$ and $\mathrm{X}$ bands.

Fig. 2-B shows the two-tones analysis of the radar $\mathrm{RX}$ section for the $\mathrm{S}$ and $\mathrm{X}$ bands. The graph shows the values of the first harmonic and of the third-order intermodulation distortion. The level of the thermal noise of the PD is also reported. The RF-to-IF conversion loss in $\mathrm{X}$ band turns out to be $59 \mathrm{~dB}$, with a spurious-free dynamic range (SFDR) of 99 $\mathrm{dB} \cdot \mathrm{Hz}^{2 / 3}$. For the $\mathrm{S}$ band instead, the conversion loss is $55 \mathrm{~dB}$, and the SFDR results to be $100 \mathrm{~dB} \cdot \mathrm{Hz}^{2 / 3}$. For the sake of comparison, we consider the conversion loss values reported in [2] since, to the best of our knowledge, that work is the only microwave-photonics RF transceiver in SOI presented so far that reports such analysis. There, the declared conversion loss ranges from 40 to $60 \mathrm{~dB}$ for signals in the frequency interval from 2 to $10 \mathrm{GHz}$, which is comparable with the values found here, and are thus typical of a microwave photonic transceiver in SOI. Although such value is larger than the typical conversion losses of standard RF systems, these systems have an unprecedented frequency flexibility, confirmed also by the similar figures for the two analyzed bands. The high values of conversion loss are due to a number of factors, the main of which are the PIC optical losses, the power of the laser limited by the two photon absorption in the silicon waveguide, and the high $\mathrm{V} \pi$ (i.e., the halfwave voltage) of the modulator. 
To test the actual radar functionality a fiber-based radar target emulator has been exploited, as represented in Fig. 1. The optical signal is extracted from the radar TX section of the PIC through the monitoring GC, delayed in an optical fiber spool of $1 \mathrm{~km}$ length, and sent to an external PD (with performance similar to the integrated one). After RF-filtering the radar signal at the desired frequency, a cascade of low-noise amplifiers (LNAs) boosts the signal, and a variable attenuator (VA) allows varying the RF power at the input of the radar RX section in the PIC. The radar subsystem has been thus characterized in terms of sensitivity, i.e., measuring the signal-to-noise ratio (SNR) of the cross-correlation between the downconverted received echo signal and the transmitted one. The test is carried out using $2 \mu$ s-long linearly chirped pulses with a bandwidth (BW) of $100 \mathrm{MHz}$ and integrating the echoes over a $20 \mathrm{~ms}$ period. As reported in Fig. $2-\mathrm{C}$, considering a minimum acceptable SNR of $10 \mathrm{~dB}$, the minimum detectable echo power is $-52 \mathrm{dBm}$ and $-38 \mathrm{dBm}$ for the $\mathrm{S}$ and $\mathrm{X}$ bands respectively. It is worth to notice that the echo power values reported here are those directly measured at the receiver input of the radar PIC, without considering any electrical front-end; therefore, the radar sensitivity including the amplification stages (as it is usually the case) would be well below $-110 \mathrm{dBm}$ assuming a front end with 60 $\mathrm{dB}$ gain and $7 \mathrm{~dB}$ noise figure as in [1], allowing to reach the performance of conventional receivers.

The radar transceiver has also been tested as an electrical communication system. A54Mbit/s 64-QAM orthogonal frequency division multiplexing (OFDM) communication signal has been generated with a vector signal generator and used a signal analyser to receive it and measure the system performance. Fig. 3 summarizes the results obtained for the OFDM signal transmission and reception in S- and X-bands. Since the generator had a carrier frequency limited to 6 $\mathrm{GHz}$, the down-conversion measure has been carried out only in the S-band.

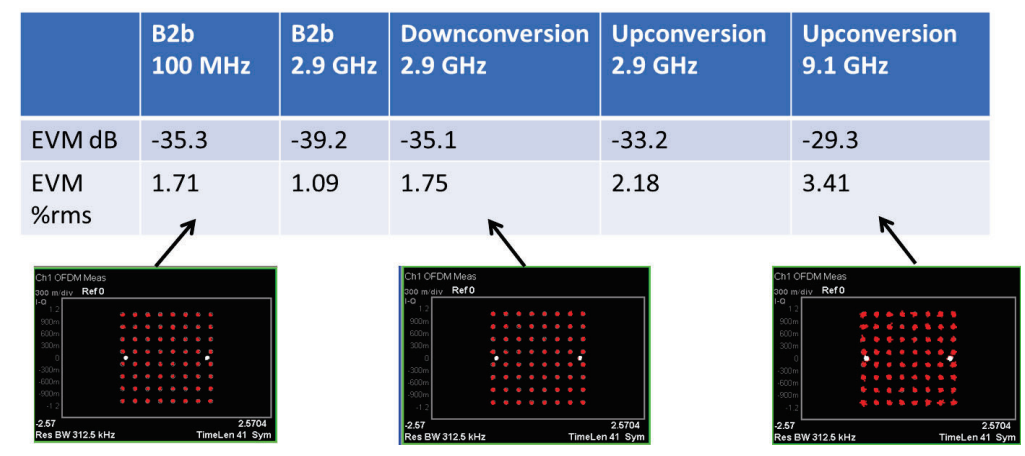

Figure 3. Electrical communications performance of the radar transceiver

\section{CHARACTERIZATION OF THE LIDAR SUBSYSTEM}

As depicted in Fig. 1, to test the lidar subsystem the output of the PIC, i.e. the signal to be transmitted, is delayed by a spool of optical fiber and an Erbium-doped fiber amplifier (EDFA) boosts the signal before it is launched in free space by a collimator. The reflected signal is collected by a second collimator and sent back to the PIC, where it is routed to the H90. Moreover, a polarization scrambler assures that the experiment is polarization independent. The employed modulation of the signal is an ON/OFF keying (OOK) pseudo-random bit sequence (PRBS) of $2^{15}-1 \mathrm{bits}$ at $170 \mathrm{Mbit} / \mathrm{s}$.

The lidar subsystem is first characterized in terms of sensitivity, and to avoid fluctuations the EDFA output is connected directly to the lidar RX GC in back-to-back configuration, and a variable optical attenuator allows to precisely set the received power. Fig. 4-A shows the SNR of the cross-correlation between the received signal and the transmitted PRBS, as a function of the optical power at the lidar RX input. The minimum detected power is $-66 \mathrm{dBm}$, considering a minimum acceptable SNR of $10 \mathrm{~dB}$. The maximum achievable SNR is about $25 \mathrm{~dB}$, which corresponds to the pedestal of the cross-correlation function for the employed waveform [5].

A demonstration of the lidar range measurement in free space is reported in Fig. 4-B, with the target is placed about 3 meters from the collimators. The target (blue curve) is correctly detected by the system 3 meters farther with respect to the back-to-back fiber loop only (orange curve). The lidar resolution is about $1 \mathrm{~m}$, and it is given by the bit rate of the signal (i.e. by its bandwidth). The measurement therefore confirms the lidar ranging functionality. In our experiment, the bit rate is limited by the lack of a DC-coupled biasing circuit for the PDs, the BW of which is therefore limited to few hundreds MHz. Once the PIC will be packaged and driven by a custom designed biasing circuit, the lidar resolution 
would be limited by the MZM to $18 \mathrm{GHz}$, with a corresponding resolution of about $1 \mathrm{~cm}$ that fits the current requirements of lidars for automotive applications [6].
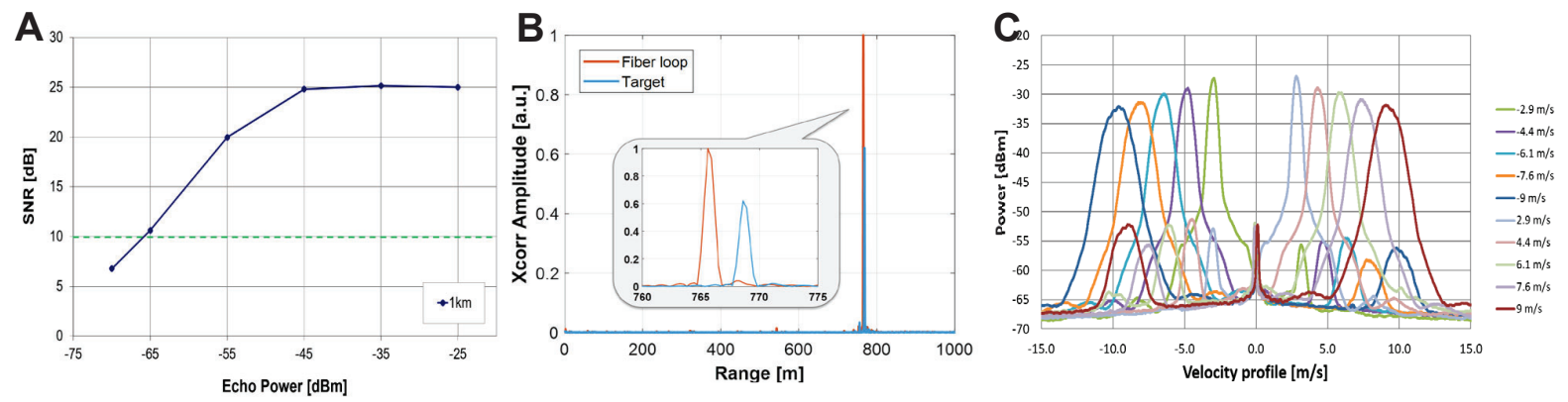

Figure 4. A) Sensitivity of the Lidar subsystem; B) Free-space range measurements of the lidar subsystem; C) Velocity profile changing the nominal speed of the target.

Using a rotating disk running at a controlled speed (with the rotation axis perpendicular to the optical beam direction), it is possible to test the velocity measurement capability of the lidar subsystem. In this case, the Doppler frequency shift is:

$$
\text { (1) } \Delta f=2 \frac{\Delta v}{c} f_{0} \sin \theta
$$

with $\Delta \mathrm{f}$ the frequency shift, $\Delta v$ the speed of the target, $\mathrm{c}$ the speed of light in vacuum, $\mathrm{f}_{0}$ the laser frequency, and $\theta$ the angle between the center of the motor and the point where the laser beam hits the edge of the disk. Thanks to the optical coherent detection scheme implemented in the PIC, it is possible to recover the frequency difference between the lidar signal reflected by the target (hence, subject to a Doppler frequency shift) and the transmitted signal at the reference frequency, revealing the target speed. Fig. 4-C shows the results of velocity measurements in the case $\theta=90^{\circ}$ (maximum detected speed). The peak of the measured curves always correctly identifies the rotation velocity of the target, with a nominal Doppler resolution of $0.5 \mathrm{~m} / \mathrm{s}$ that is essentially determined by the integration time. This resolution matches with the one requested for the high-demanding navigation systems [7]. We can see that the peak width grows with the target speed: this is due to the increased vibrations of the target [8] that cause a spreading of the $\theta$ angle. Moreover, it is interesting to note that both positive and negative velocities can be correctly measured, thanks to the coherent detection. Finally, we can notice that a good extinction $(>20 \mathrm{~dB})$ of the image frequency in the Doppler profile is achieved due to the high quality of the integrated $90^{\circ}$ hybrid coupler in the PIC.

The software defined lidar system has been also tested as free-space optical communication system. The chip is used to transmit the optical data signal that is sent to the free-space link through the Tx collimator. Then, the signal is collected from the Rx collimator and detected with an external receiver connected to the error analyser for the bit error rate (BER) measure and to the sampling scope for eye diagram measure. Both the on-off keying (OOK) modulation at $12.5 \mathrm{Gbit} / \mathrm{s}$ and differential phase shift keying (DPSK) modulation at $9.95 \mathrm{Gbit} / \mathrm{s}$, more robust to channel attenuation variation, have been verified.

The bit error rate (BER) measurements are reported in Fig. 5 for OOK (left) and DPSK (right) signals respectively and compared with the ones obtained with an external Lithium Niobate (LiNbO3) modulator. In the OOK case we can observe a penalty of $1 \mathrm{~dB}$ due to the higher Vpi of the SOI modulator compared to the LiNbO3 one. In the DPSK case when the SOI modulator is used in single drive configuration we have $1 \mathrm{~dB}$ penalty compared to the LiNbO3, while when it is used in dual drive configuration the performance are $1 \mathrm{~dB}$ better than LiNbO3. The received eye diagrams are also shown in the insects.

\section{COMMENTS AND CONCLUSIONS}

In this paper, we have presented the first realization of a photonic-integrated combined radar\&lidar system in SOI that can also been exploited for RF/optical communications. The PIC includes in a single ultra-compact integrated device a frequency-flexible radar/RF communication transceiver, and an advanced lidar/optical communication transceiver with coherent photonic detection for range and velocity measurement.

Here we have presented the results of the tests that have been run separately on the radar and lidar subsystems directly 
on the bare PIC. The tests clearly show, on the one hand, the very large frequency flexibility and good sensitivity and linearity of the PIC radar subsystem, and on the other hand, the resolution in both range and velocity of the PIC lidar subsystem. In conclusion, the breakthrough PIC architecture presented here combining radar\&lidar functionalities in a single tiny device, shows potential for enabling a new category of surveillance devices for complex observations in complex environments.

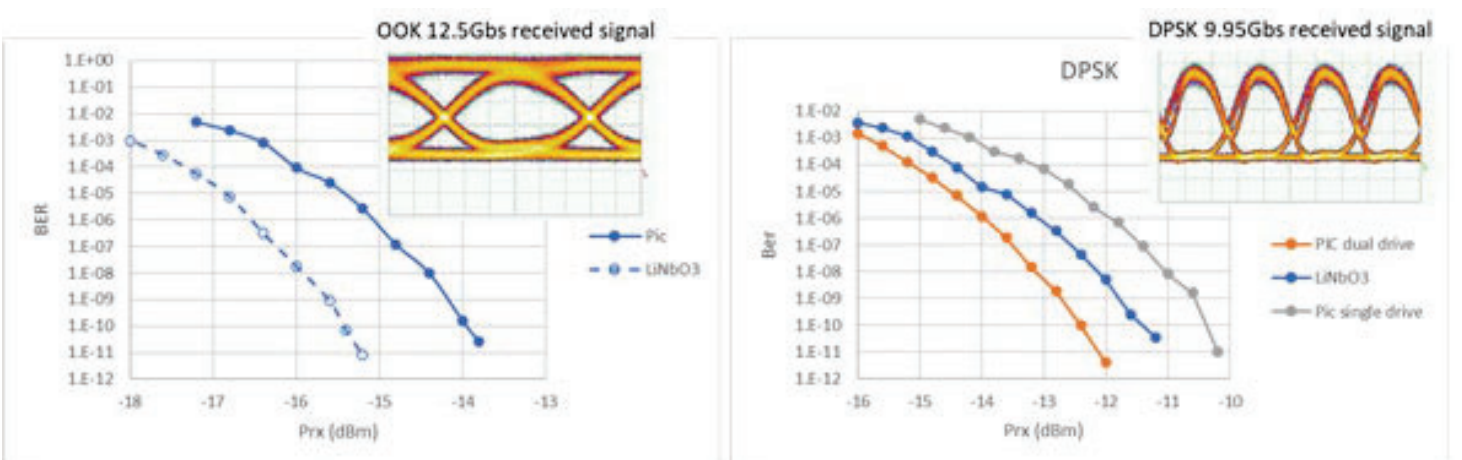

Figure 5. (left): BER measurements of the lidar system used as free-space optical communication system using OOK $12.5 \mathrm{Gbit} / \mathrm{s}$ signals compared with the performance using a commercial LiNbO3 modulator. (Right): BER measurements in case of DPSK $9.95 \mathrm{Gbit} / \mathrm{s}$ signal driving the PIC modulator in single and dual drive configuration, compared with the performance using a commercial $\mathrm{LiNbO} 3$ modulator The received eye-diagrams are reported in the insets.

\section{ACKNOWLEDGMENT}

This work has been funded by the Italian Space Agency under the project RODI, contract number 2017-33-H.O, CUP: F52F17000830005.

\section{REFERENCES}

[1] P. Ghelfi et al., “A fully photonics-based coherent radar system,” Nature, vol. 507, no. 7492, pp. 341-345, Mar. 2014, doi: 10.1038/nature13078.

[2] M. Chen, H. Yu, B. Yang, Y. Li, H. Chen, and S. Xie, "A silicon integrated microwave-photonic transceiver," in 2017 Optical Fiber Communications Conference and Exhibition (OFC), Mar. 2017, pp. 1-3.

[3] J. K. Doylend and S. Gupta "An overview of silicon photonics for LIDAR", Proc. SPIE 11285, Silicon Photonics XV, 112850J (26 February 2020);

[4] F. Scotti, D. Onori, M. Scaffardi, E. Lazzeri, A. Bogoni, and F. Laghezza, "Multi-Frequency Lidar/Radar Integrated System for Robust and Flexible Doppler Measurements," IEEE Photon. Technol. Lett., vol. 27 , no. 21, pp. 2268-2271, Nov. 2015, doi: 10.1109/LPT.2015.2461458.

[5] Hochberg, Michael, et al. "Silicon photonics: the next fabless semiconductor industry." IEEE Solid-State Circuits Magazine 5.1 (2013): 48-58.

[6] Halir, Robert, et al. "High-performance 90 hybrid based on a silicon-on-insulator multimode interference coupler." Optics letters 36.2 (2011): 178-180.

[7] P. Adany, C. Allen, and R. Hui, "Chirped Lidar Using Simplified Homodyne Detection," Journal of Lightwave Technology, vol. 27, no. 16, pp. 3351-3357, Aug. 2009, doi: 10.1109/JLT.2009.2016220.

[8] X. Sun and J. B. Abshire, "Comparison of IPDA lidar receiver sensitivity for coherent detection and for direct detection using sine-wave and pulsed modulation," Opt. Express, vol. 20, no. 19, p. 21291, Sep. 2012, doi: 10.1364/OE.20.021291. 